\title{
Possibilités d'utilisation des procédés à membranes pour la valorisation des produits de la mer et dans l'aquaculture
}

\author{
par \\ P. BELLEVILLE et F. QUEMENEUR
}

\section{R és u m é}

Différentes applications des procédés de séparation par membranes semi-perméables (ultrafiltration, électrodialyse, microfiltration, osmose inverse...) se rapportant aux produits de la mer sont d'abord passées en revue. Ces applications sont classées en trois catégories : la concentration et le fractionnement de matières organiques dissoutes ou en suspension dans l'eau de mer, l'extraction de certains constituants d'algues, le traitement de concentrés protéiques et d'hydrolysats de poissons.

D'autres possibilités d'utilisation sont ensuite envisagées, notamment pour la purification de l'eau de décontamination des coquillages et des bassins d'aquaculture en circuit fermé et pour le traitement des eaux de lavage de poissons dans les ports.

Dans l'ensemble, l'application de ces techniques n'a pas encore connu le développement espéré il y a une dizaine d'années.

\section{S u m m a r y}

POSSIBILITIES OF USING PROCESSES FOR VALORIZING SEA PRODUCTS AND IN AQUACULTURE

Different applications for separating processes through semi permeable membranes (ultrafiltration, electro-dialysis, microfiltration,

Institut Universitaire de Technologie, Département génie chimique, B.P. 420 44606 Saint-Nazaire cedex. 
reverse osmosis...) related to sea products are first reviewed. These applications are classified into three categories: concentration and fractioning of organic materials dissolved or suspended in sea water, extraction of certain algae constituents, treatment of fish protein concentrate and fish hydrolysates.

Then other possible uses are considered, particulary for purifying decontamination water of shell and closed circuit aquaculture basins and for treating fish washing water in ports.

On the whole, applications of these techniques have not yet been developed as was expected about ten years ago.

\section{INTRODUCTION}

Les procédés de séparation par membranes semi-perméables (osmose inverse, ultrafiltration, électrodialyse, microfiltration...) ont donné lieu ces quinze dernières années à de nombreuses applications ou tentatives d'utilisation se rapportant au milieu marin et à l'aquaculture, d'une part dans le but d'acquérir une meilleure connaissance du milieu, d'autre part pour valoriser les produits ou sous-produits issus de ce milieu.

Nous nous contenterons d'évoquer dans cet exposé l'utilisation de l'osmose inverse pour le dessalement de l'eau de mer ou des eaux saumâtres qui représente de loin l'application la plus importante des membranes dans ce milieu, tant pour l'étendue des surfaces mises en œuvre que par les volumes traités.

Nous développerons surtout les applications ayant trait aux produits d'origine végétale ou animale issus du milieu marin.

Nous passerons d'abord en revue quelques applications déjà signalées dans la littérature scientifique (ou dans la presse) telles que :

- la concentration et le fractionnement des matières organiques dissoutes et du phytoplancton;

- l'extraction de composants spécifiques d'algues;

- l'hydrolyse enzymatique de concentrés protéiques de poissons et l'extraction d'acides aminés des hydrolysats.

Nous examinerons ensuite d'autres possibilités d'utilisation des membranes qui nous semblent intéressantes, notamment dans le domaine de la purification de l'eau (décontamination de coquillages, aquaculture en circuit fermé) et celui du traitement des saumures et des eaux de lavage de poissons. 


\section{APPLICATIONS EXISTANTES}

\subsection{Concentration et fractionnement de matières organiques dissoutes ou en suspension}

De nombreuses utilisations de l'ultrafiltration, essentiellement à des fins analytiques, sont signalées dans ce domaine dans la littérature.

Dès 1968, Barber a utilisé une cellule d'ultrafiltration équipée d'une membrane DIAFLO à seuil de coupure 500 pour concentrer les substances organiques contenues dans l'eau de mer à différentes profondeurs $(50,1200$ et $5000 \mathrm{~m})$ et en déduire un profil vertical de distribution des matières organiques dissoutes.

En 1974, Ogura a procédé, après une filtration préalable sur membrane à $0,45 \mu \mathrm{m}$, au fractionnement de la matière organique dissoute dans une eau de mer côtière par ultrafiltration sur membranes DIAFLO UM-05, UM-10 et UX-100, ce qui lui a permis de connaître la distribution suivant les masses molaires des substances organiques dissoutes.

En 1977, une équipe russe (Baturina et coll.) a utilisé une technique sensiblement identique pour comparer les distributions des matières organiques dissoutes dans des eaux de mer équatoriales prélevées à quelques dizaines de mètres de profondeur avec celles prélevées entre 1000 et $3000 \mathrm{~m}$.

En 1979, Boonlayangoor et coll. ont déterminé grâce à l'ultrafiltration les variations saisonnières des teneurs en phytoplancton dans le lac Michigan.

Plusieurs auteurs (Gnassia-Barelli et coll. 1977, 1978 et 1980, Gachter et coll. 1978, Mc Knicht, 1982) se sont servis de l'ultrafiltration (cellule Amicon avec membranes à seuils de coupure 10000 , 2000 et 500) pour fractionner les produits sécrétés par le phytoplancton en présence de cuivre dissous et évaluer leur pouvoir complexant vis-à-vis de ce métal.

Une autre utilisation de l'ultrafiltration est signalée (Picer et coll., 1978) dans le cadre d'une étude de l'action de dérivés organo-chlores sur une culture de phytoplancton en laboratoire.

En dehors de l'aspect analytique, l'ultrafiltration et la microfiltration peuvent être utilisées pour la concentration de phytoplancton en vue de fournir aux écloseries et nurseries de crustacés ou poissons une eau enrichie en éléments nutritifs.

Des essais ont été réalisés dans ce sens en laboratoire ou sur de petites unités (écloseries de l'Ile d'Yeu et de Houat par exemple). Mais à notre connaissance, ces essais n'ont pas été suivis de réalisations à grande échelle. 
Les professionnels évoquent la complexité de la mise en œuvre des techniques à membranes ainsi que les coûts d'investissement et de fonctionnement sans commune mesure avec les volumes relativement modestes d'eau à traiter.

Les " concentrats» de phytoplancton obtenus en tant que " sousproduits » de la purification d'eaux de lagunes par ultrafiltration ou microfiltration peuvent par contre trouver des débouchés intéressants pour l'élevage des huîtres ou autres coquillages. Des essais dans ce sens, en cours à l'université de Montpellier, semblent prometteurs (Rumeau, 1983).

Les techniques à membranes peuvent également aider efficacement à la récupération de l'énergie solaire à partir de la biomasse aquatique.

Une illustration spectaculaire en a été présentée par Grégor (1978) qui décrit un prototype (fig. 1) pour la culture de micro-algues à combustibles faisant intervenir trois techniques à membranes différentes : l'ultrafiltration, l'extraction par solvant et l'électrodialyse (on pourrait éventuellement y adjoindre la microfiltration et l'osmose inverse).

Les algues dont la teneur dans le bassin de culture est de l'ordre de 0,1 à $0,2 \mathrm{~g} / 1$ sont concentrées à plus de $10 \mathrm{~g} / 1$ par ultrafiltration pour cette étape avant d'être introduites dans la cuve de fermentation méthanique. Les sous-produits de la fermentation sont extraits à l'aide d'une autre membrane d'ultrafiltration et passent à travers une cellule de dissociation d'eau dans laquelle se séparent $\mathrm{CO}_{2}$ et $\mathrm{NH}_{3}$ d'une part, acides et bases d'autre part.

Une partie du gaz carbonique dissous dans un courant basique est renvoyée dans le fermenteur tandis que l'excédent est recyclé avec l'ammoniac dans le réservoir de culture d'algues.

Les acides organiques qui ont une valeur commerciale sont récupérés par extraction par solvant à travers une membrane. Enfin l'électrodialyse permet de concentrer les sels minéraux résiduels et de récupérer de l'eau pure.

\subsection{Extraction de compososants spécifiques d'algues}

L'utilisation de l'ultrafiltration pour l'extraction de substances intéressantes dans les algues broyées est signalée dans un brevet dès 1972 (Strong C.H.). Il s'agissait de purifier et concentrer des solutions aqueuses de polysaccharides extraites d'algues marines.

Plus près de nous, la Société Goémar de Saint-Malo a préparé par ultrafiltration d'algues broyées et décantées ce qu'elle appelle un «filtrat physiologique d'algues " (Le Figaro, 1983) qui pourrait être utilisé directement en physiothérapie ou servir de base à des 


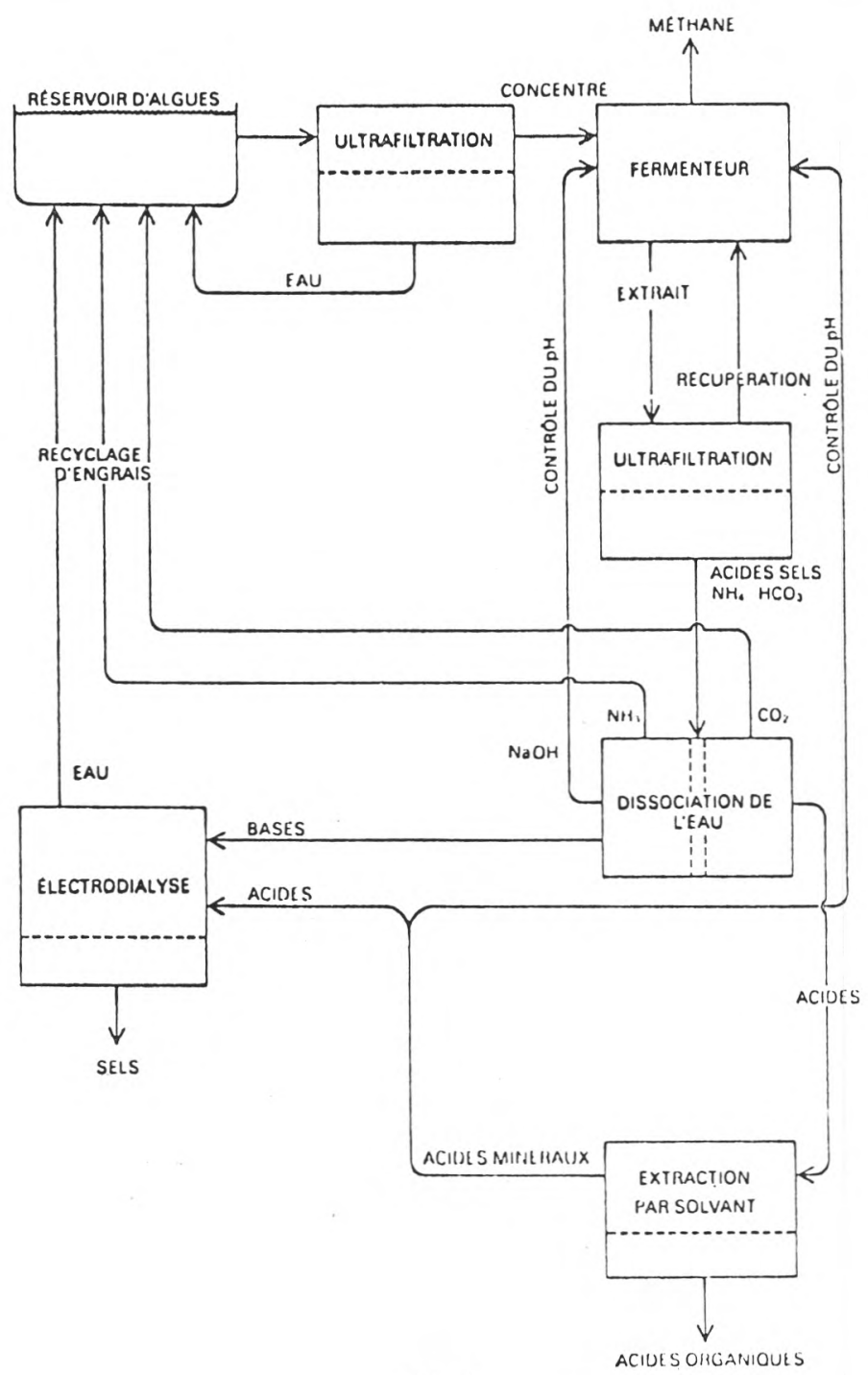

fig. 1

La culture d'algues à combustible. - Algae culture producing fuel.

« boissons de santé ». Malheureusement (pour notre exposé!) la Société Goémar a refusé de nous communiquer le moindre renseignement sur la technique mise en œuvre et sur les perspectives de développement du procédé. 


\subsection{Traitement des concentrés protéiques et des hydrolysats de poissons}

Les protéines de poissons généralement obtenues à partir d'espèces non commercialisées ou de déchets représentent des potentialités importantes pour l'alimentation humaine (Durand et Lagoin, 1983). L'utilisation judicieuse des techniques à membranes devrait permettre de valoriser ces produits soit en aidant à les présenter sous une forme soluble plus facilement assimilable par l'organisme, soit en facilitant la récupération d'acides aminés naturels utilisables dans l'industrie pharmaceutique par exemple.

Une méthode souvent utilisée pour l'obtention de concentrés protéiques de poissons consiste à broyer les poissons puis à extraire l'eau et les lipides par l'isopropanol. Cette méthode conduit, après séchage, à une poudre contenant environ $85 \%$ de protéines. Mais ces protéines sont pratiquement insolubles et difficiles à disperser dans l'eau, ce qui limite considérablement la possibilité de leur utilisation pour l'alimentation humaine.

La solubilisation des protéines de poissons peut se faire de différentes façons : par voie chimique (hydrolyse acide ou traitement par des solutions salines ou alcalines) ou par voie enzymatique naturelle ou en réacteur.

Les procédés chimiques présentent divers inconvénients en particulier la destruction de certains acides aminés et la nécessité d'une neutralisation finale entraînant une augmentation de la teneur en sels du produit. L'électrodialyse est parfois utilisée pour remédier à cet inconvénient (Kinumaki, 1978).

L'hydrolyse enzymatique ne présente pas ces défauts mais pose d'autres problèmes, notamment la lenteur du processus ou le coût des enzymes si l'on cherche à être plus performant.

Le recyclage des enzymes en réacteur à membranes d'ultrafiltration permet à la fois d'accroître la vitesse d'hydrolyse et de réduire le coût lié à l'apport d'enzymes.

C'est la méthode mise en ouvre par Cheftel (1971) qui est illustrée par la figure 2 .

La cellule d'ultrafiltration utilisée est équipée d'une membrane à seuil de coupure 10000 et la masse molaire de l'enzyme (la pronase) comprise entre 19000 et 35000 . Cette enzyme est donc retenue dans le réacteur (en même temps que les plus grosses molécules de protéines), tandis que les peptides formées ainsi que les produits de la solubilisation qui inhibent la réaction passent à travers la membrane.

Malheureusement dans ce procédé les bactéries sont également recyclées et les essais réalisés n'ont pu aller au-delà de quatre jours en raison du développement bactérien dans le milieu. 


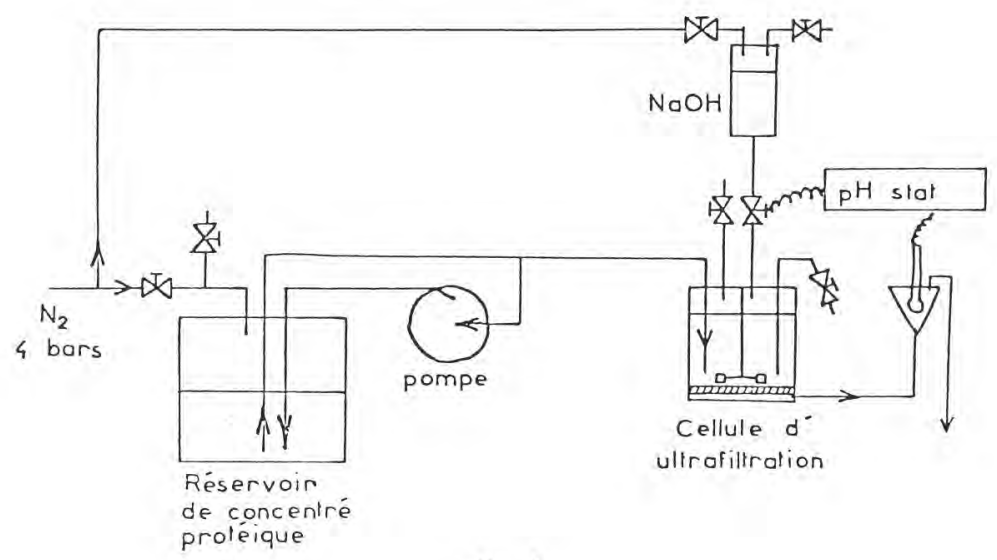

fig. 2

Solubilisation continue de concentré protéique de poisson par la pronase.

Continuous solubilization of fish protein concentrate with pronase.

Un autre créneau d'utilisation des techniques à membranes se situe au niveau de l'extraction d'acides aminés lévogyres (L. aminoacides) à partir du liquide clair issu de la séparation des principales protéines et protides des hydrolysats de poissons.

Cette application fait l'objet d'un brevet français (Dubois et Hache, 1977) qui considère l'exemple d'un hydrolysat de viscères et de poissons non commercialisés, dits «faux poissons ", préparé à bord du bateau de pêche selon un procédé enzymatique et aussitôt stabilisé par pasteurisation à $90^{\circ} \mathrm{C}$ pendant $1 \mathrm{~h}$ en milieu acide citrique.

Cet hydrolysat est centrifugé à terre et la phase liquide contenant des pigments colorés, des protéines, des polypeptides, des acides aminés lévogyres libres et des sels, en particulier du chlorure de sodium, est traitée dans l'installation représentée par la figure 3 .

Introduite dans la cuve 1 la phase liquide passe dans l'ultrafiltre 2 équipé de membranes à seuil de coupure pouvant être compris entre 5000 et 25000 afin d'éliminer les protéines et les peptides risquant de fermenter en présence de bactéries apportées par l'air. En fin d'opération le concentrat est traité selon les procédés classiques pour en extraire les protéines.

Le perméat est stocké dans la cuve 6 et analysé pour en connaître la teneur en protéines et protides. Si elles sont présentes en quantité notable, ces protéines sont précipitées par addition de laurylsulfate de sodium dans la cuve 7 et filtrées sur membranes à $0,22 \mu \mathrm{m}$ en $7 \mathrm{a}$, le filtrat étant dirigé sur le réservoir 11. En cas d'absence de pro- 


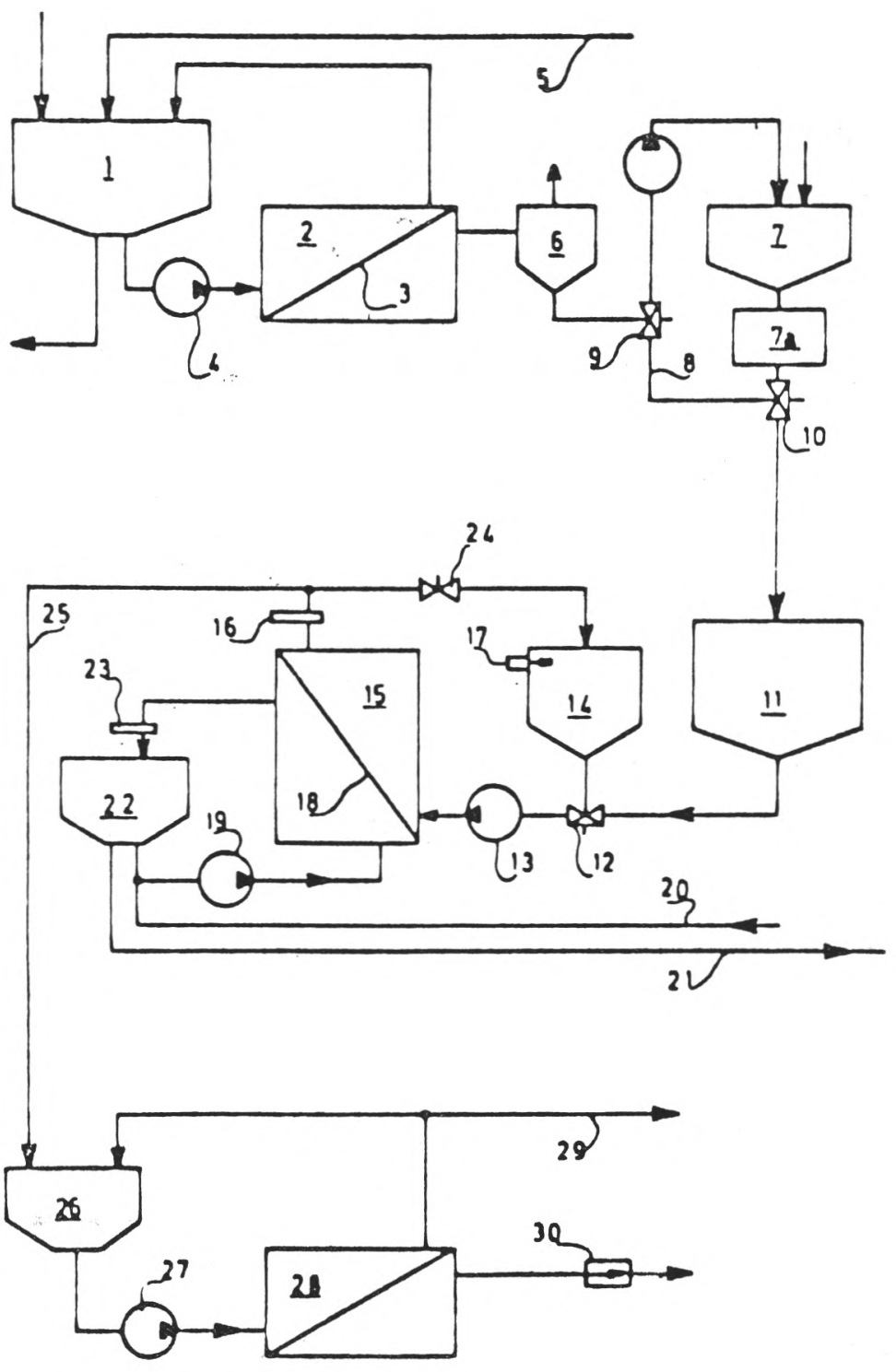

fig. 3

Extraction d'acides aminés dans les hydrolysats de poissons. Extraction of amino acids in fish hydrolysate. 
téines le perméat passe directement de 6 en 11, d'où il est introduit dans le circuit d'électrodialyse. La sonde 16 de conductivité permet de suivre l'appauvrissement en sel dans le circuit. On arrête l'électrodialyse quand la teneur en sels est de $100 \mathrm{ppm}$.

La solution d'acides aminés ainsi déminéralisée est alors dirigée dans la cuve 26 et peut être concentrée jusqu'à $66 \mathrm{~g} / 1$ par osmose inverse avant déshydratation.

Dans un domaine assez proche nous avons relevé une application de l'ultrafiltration pour le fractionnement de l'ATP ase issu d'hydrolysats de muscle d'otarie (Tamiya et coll., 1977).

Signalons enfin les essais d'ultrafiltration effectués aux U.S.A. (Hood et coll., 1976; Joh et Hood, 1979) sur des eaux de lavage de clams émincés pour en extraire des arômes et agents de sapidité naturels particulièrement intéressants. Malheureusement l'ultrafiltration a conduit dans ce cas à des propriétés organoleptiques inférieures à celle que l'on peut obtenir par évaporation sous vide.

\section{Autres possibilités d'utilisation des membranes}

Les techniques à membranes, plus particulièrement l'ultrafiltration, pourraient trouver d'autres applications dans le domaine de l'aquaculture et des produits de la mer notamment au niveau de la purification de l'eau et du traitement des saumures et des eaux de lavage de poissons.

\subsection{Purification de l'eau de décontamination des coquillages et des bassins d'aquaculture en circuit fermé}

En ce qui concerne la décontamination des coquillages, notamment des huîtres ayant pu, du fait de la proximité d'émissaires d'eaux usées, retenir des germes pathogènes, une méthode courante consiste à utiliser de l'eau chlorée. Or au contact de l'eau chlorée les mollusques se ferment hermétiquement et on ne décontamine que les coquillages.

Par contre placés dans une eau bactériologiquement pure les coquillages relarguent les germes pathogènes. Le problème est alors de se procurer cette eau stérile et de piéger les germes libérés. Nous pensons que l'ultrafiltration, sur membranes appropriées, est un moyen compétitif pour résoudre ce problème.

L'aquaculture en circuit fermé nécessite également un traitement continu de l'eau injectée dans le circuit, la chaîne de traitement comporte généralement une étape de décantation lamellaire, une dénitrification sur filtre bactérien et une stérilisation.

Les techniques de stérilisation les plus courantes sont les radiations ultraviolettes, l'ozonation et la chloration. Toutefois les deux dernières techniques, très utilisées pour le traitement des eaux potables, ne sont pas toujours transposables en aquaculture car 
elles produisent, surtout en eau de mer, des composés toxiques pour l'élevage. En revanche, le rayonnement UV ne présente pas cet inconvénient et il est donc couramment utilisé. Il implique cependant d'avoir une eau très peu chargée en matière en suspension et une faible teneur en fer (Liao, 1980; Maurel, 1983).

Là encore, il semble que l'ultrafiltration soit une technique de stérilisation intéressante : elle peut en effet retenir tous les germes ichtyo-pathogènes y compris les virus, et le risque de toxicité est nul.

\subsection{Traitement des eaux de lavage de poissons dans les ports}

Ces eaux contiennent, outre des matières en suspension, des substances dissoutes (souvent riches en protéines). Les techniques à membranes (microfiltration et ultrafiltration notamment) peuvent là encore s'avérer intéressantes en associant épuration et récupération des produits valorisables.

\section{CONCLUSION}

Les procédés de séparation par membranes semi-perméables ont donné lieu à de nombreuses utilisations ou essais d'utilisation pour le traitement et la valorisation des produits de la mer.

Parmi ces procédés c'est l'ultrafiltration qui s'applique le plus souvent lorsqu'il s'agit de concentration ou de fractionnement de produits ou de réacteur enzymatique à membrane.

Si l'on fait abstraction de la préparation d'eau pure, l'osmose inverse trouve son créneau dans la concentration de petites molécules dissoutes et l'électrodialyse dans le dessalement des solutions protéiques provenant des hydrolysats de poissons. Quant à la microfiltration, elle pourrait être davantage utilisée pour la concentration du phytoplancton et autres micro-algues.

Mais dans l'ensemble, l'application de ces différentes techniques n'a pas connu le développement que certains auteurs prévoyaient il y a une dizaine d'années (Porter, 1971).

Cela nous semble dû à une connaissance insuffisante des possibilités de ces techniques dans le milieu concerné, à la nécessité d'investissements relativement élevés, à la crainte de coûts de fonctionnement élevés au niveau du changement des membranes.

On peut penser que l'abaissement de ces coûts (notamment celui des membranes minérales) permettra à ces techniques d'occuper à l'avenir une place plus importante dans le traitement des produits de la mer. 


\section{Bibliographie}

BARBER (R. T.) (1968). - Dissolved organic carbon from deep waters resists microbiol. Oxydation. Nature, 220, october, 274-275.

Baturina (M. V.), LJutsarev (S. V.), Romankevich (E. A.) (1977). - Fractionning by ultrafiltration the organic mater of the marine water. Pol. Arch. Hydrobio., 24, 99-102.

Boonlayangoor (C.), Allen (H. E.), Noll (K E.) (1979). - Colloid in Lake Michigan water: nature, size distribution, seasonnal variationand reaction with metals. Report I.T.T. Chicago, July, 227 p.

Cheftel (C.), Ahern (M.), Wang (D.), Tannenbaum (S.) (1971). - Solubilisation enzymatique continue du concentré protéique de poisson. Agr. Food Chem., $19,155$.

Dubois (E.), Hache (J.) (1977). - Purification de matières organiques en vue de la production des acides aminés. Brevet français $\pi^{\circ} 2399213,7 \mathrm{p}$.

Durand (P.), Lagoin (Y.) (1983). - Valorisation des sous-produits de la pêche. Réalisations et perspectives. Bull. Inf. ISTPM, 330, 20 p.

GACHTER (R.), DAVIS (J. S.), MARES (A.) (1978). - Regulation of Copper availability to phytoplankton by macro-molecules in lake water. Environ. Sci. Technol,, 12 (13), 1416-1421.

Gnassia-Barelli (M.), Romeo (M.), Laumond (E.), Pesando (D.) (1977). - Techniques d'ultrafiltration. Applications à la mise en évidence de complexants des métaux produits par le phytoplancton. Rev. Int. Océanogr. Méd., 48, 131-135.

Gnassia-Barelli (M.), Romeo (M.), Laumond (E.), Pesando (D.) (1978). - Experimental studies on the relationship between natural copper complexes and their toxicity to phytoplankton. Marine Biology, 47, 15-19.

Gnassia-Barellit (M.), Pesando (D.), Laumond (F.) (1980). - Recherche de complexants naturels de métaux-traces en particulier du cuivre provenant du phytoplancton. Actual. Biochim. Mar., 2, 89-102.

GREGoR (H. P.), GREGOR (C. D.) (1978). - Les applications des membranes synthétiques. Pour la Science, 11, 73-89.

Hoon (L. F.), ZALL (R. R.) CONWAY (R. L.) (1976). - Conversation of minced clam wash water into clam juice: waste brandling or produit development? Food Produit Devel., 10 (10), 86-88.

JoH (Y.), Hood (L.F.) (1979). - Preparation and properties of deshydrated clam flavor clam processing wash water. J. Food Sci, 44 (6), 1612-1614.

KINUMAKI (T.) (1978). - Possibilities for the production and application of lignefied fish protein in the IPFC area. Proc. IFPC, 18 (3), 492-506.

LiaO (P. B.) (1981). - Treatments units used in reniculation systems for intensive aquaculture. Proc. World Symp. on Aquaculture in Heated effluents and reniculation systems, Stavanger, 28-30 May 1980, vol. 1, Berlin, 1981.

Le Figaro (10-11 décembre 1983). - Goëmar : les dons de la mer.

Maurel (P.) (1983). - Les circuits fermés en aquaculture. Technologie et domaine d'application. La Pisciculture Française, 71, 5-18.

Mc KNight (D.) (1981). - Chemical and biological process Controlling the response of a freshwater ecosystem to copper stress: a field study of the $\mathrm{Ca} \mathrm{SO}_{4}$ treatment of Mill Pond Reservoir, Burlington, Massachusetts. Limnol. Oceanogr., 26 (3), 518-531.

OGURA (N.) (1974). - Molecular weight fractionation of dissolved organic matter in coastal seawater by ultrafiltration. Marine Biology, 24, 305-312. 
Picer (M.), Picer (N.) ,Krsnic (F.), Sipos (V.) (1978). - Investigation of the fate of some chlorimated hydrocarbons in laboratory grown phytoplankton culture. Quatrièmes Journées d'études sur les pollutions marines en Méditerranée. Antalya (Turquie), $24-27$ nov. Publ. I.C.S.E.M. Monaco, 1979, 453-456.

Porter (M. C.), Michaels (A. S.) (1971). - Membrane applications. Part. 3: Applications in the processing of meat by products. Chem. Tech., July, 440-445.

Rumeau (M.) (1983). - Université de Montpellier, communication personnelle.

Strong (C. H.) (1972). - Purification and concentration of an aqueous polysaccharide solution obtained from sea algae. Brevet Can. Appl. 149, 534, 16 Aug. (C.A. 1974, 81, 39358 v).

TamiYa (T.), Arai (S. M.), Tsuchiya (T.), Matsumoto (J.) (1977). - Fractionation of ATPase accelerating peptide from fur seal muscle hydrolysate. Bull. Jap. Soc. Scien. Fisheries, 43 (7), 869-876. 